Meta

Journal des traducteurs

Translators' Journal

\title{
La entonación monótona y la calidad de la interpretación simultánea: frecuencia, conceptualizaciones y efectos
}

\section{Ángela Collados Aís}

Volume 61, numéro 3, décembre 2016

URI : https://id.erudit.org/iderudit/1039224ar

DOI : https://doi.org/10.7202/1039224ar

Aller au sommaire du numéro

Éditeur(s)

Les Presses de l’Université de Montréal

ISSN

0026-0452 (imprimé)

1492-1421 (numérique)

Découvrir la revue

Citer cet article

Collados Aís, Á. (2016). La entonación monótona y la calidad de la interpretación simultánea: frecuencia, conceptualizaciones y efectos. Meta, 61(3), 675-691. https://doi.org/10.7202/1039224ar
Résumé de l'article

Compte tenu du débat sur les facteurs qui contribuent à déterminer la perception de la qualité en interprétation simultanée, cet article présente une étude empirique centrée sur l'intonation comme un des facteurs déterminants dans l'évaluation de la qualité en interprétation.

Contrairement à d'autres études qui cherchent à étudier un ensemble de facteurs, celle-ci adopte une approche plus individualisée afin de permettre une compréhension plus approfondie de l'importance accordée à l'intonation. L'intonation monotone de même que les caractéristiques et les effets que les usagers associent à l'intonation ont été analysés. Il apparaît que le facteur de l'intonation monotone est considéré très fréquent en interprétation simultanée. Pareillement, on constate deux conceptualisations fondamentales concernant les effets de l'interprétation : les effets psychologiques de l'intonation monotone chez les usagers et les effets directement liés à la transmission correcte du discours. Ces résultats viendraient confirmer le lien entre les composantes non verbales de la communication, dont l'intonation, et les composantes verbales, telles que la cohésion et la transmission correcte du discours, comme en témoigne le nombre d'articles publiés dans le domaine de la perception de la qualité chez les usagers d’une interprétation.
Ce document est protégé par la loi sur le droit d'auteur. L'utilisation des services d’Érudit (y compris la reproduction) est assujettie à sa politique d'utilisation que vous pouvez consulter en ligne.

https://apropos.erudit.org/fr/usagers/politique-dutilisation/ 


\title{
La entonación monótona y la calidad de la interpretación simultánea: frecuencia, conceptualizaciones y efectos
}

\author{
ÁNGELA COLlados Aís \\ Universidad de Granada, Granada, España* \\ colladosais@gmail.com
}

\section{RÉSUMÉ}

Compte tenu du débat sur les facteurs qui contribuent à déterminer la perception de la qualité en interprétation simultanée, cet article présente une étude empirique centrée sur l'intonation comme un des facteurs déterminants dans l'évaluation de la qualité en interprétation.

Contrairement à d'autres études qui cherchent à étudier un ensemble de facteurs, celle-ci adopte une approche plus individualisée afin de permettre une compréhension plus approfondie de l'importance accordée à l'intonation. L'intonation monotone de même que les caractéristiques et les effets que les usagers associent à l'intonation ont été analysés. Il apparaît que le facteur de l'intonation monotone est considéré très fréquent en interprétation simultanée. Pareillement, on constate deux conceptualisations fondamentales concernant les effets de l'interprétation: les effets psychologiques de l'intonation monotone chez les usagers et les effets directement liés à la transmission correcte du discours. Ces résultats viendraient confirmer le lien entre les composantes non verbales de la communication, dont l'intonation, et les composantes verbales, telles que la cohésion et la transmission correcte du discours, comme en témoigne le nombre d'articles publiés dans le domaine de la perception de la qualité chez les usagers d'une interprétation.

\section{ABSTRACT}

An empirical research was carried out in order to analyze intonation as a quality criterion in simultaneous interpreting. As opposed to previous studies on quality expectations, this paper focusses on one single criterion, aiming at finding out the discrepancies in the relative weight given to intonation between the subject's expectation pattern and the actual impact of intonation on their assessment. With this in mind, we scrutinize the most frequent level of monotony in simultaneous interpreting, as well as the effects of the interpreter's intonation on the audience when assessing simultaneous interpreting performances.

The results of this study show that monotonous intonation is common in simultaneous interpreting and suggest two basic ways of conceptualization linking the intonation to the effects on the simultaneous interpreting. On the one hand, the psychological effects of the interpreter's monotonous intonation on the audience. On the other, those effects that directly influence another quality criterion, sense consistency. The latter stresses the relation between the non verbal criterion intonation and the verbal criteria cohesion and sense consistency, which have been consistently identified as the most important ones in most of the surveys on quality expectations.

\section{RESUMEN}

Se presenta una investigación empírica realizada con el objetivo de analizar la entonación como parámetro de calidad de la interpretación simultánea. A diferencia de trabajos anteriores sobre expectativas de calidad, este estudio contempla únicamente un pará- 
metro. Se espera que este tipo de análisis más 'individualizado' proporcione explicaciones sobre la importancia divergente que se ha otorgado a la entonación en las investigaciones sobre expectativas y evaluación de la calidad de la interpretación realizadas hasta la fecha. Para ello, se analizan el nivel de monotonía más frecuente en interpretación, así como las características y efectos que los usuarios asocian con la entonación del intérprete a la hora de evaluar una interpretación simultánea.

Los resultados indican que la entonación monótona aparece con frecuencia en la interpretación simultánea. Permiten inferir, además, dos conceptualizaciones básicas vinculadas a los efectos de la interpretación. Por una parte, los efectos psicológicos que la entonación monótona del intérprete produce en los usuarios. Por otra parte, los efectos que inciden directamente sobre la transmisión correcta del sentido. Estos últimos ponen de manifiesto los vínculos que se establecen entre el parámetro no verbal entonación y los parámetros verbales cohesión y transmisión correcta del sentido, es decir, los parámetros que en la mayoría de los trabajos realizados para sondear las expectativas de calidad de los usuarios han sido señalados como los más relevantes.

\section{MOTS-CLÉS/ KEYWORDS/PALABRAS CLAVE}

interprétation simultanée, qualité en interprétation, intonation, conceptualisations, effets simultaneous interpreting, quality criteria, intonation, conceptual analysis, effects interpretación simultánea, parámetros de calidad, entonación, conceptualizaciones, efectos

\section{Planteamiento general}

Si bien hasta la fecha la investigación sobre expectativas de calidad en interpretación simultánea (IS) ha sido prolífica, la mayoría de los trabajos han seguido el esquema básico de cuestionario propuesto por Bühler (1986). Este cuestionario recogía los parámetros de calidad que teóricamente estaban llamados a contribuir a una mayor o menor calidad de la IS. Los numerosos estudios que le siguieron indagaban la participación de los distintos parámetros propuestos en el constructo calidad, incluyendo nuevos criterios o proponiendo distintas organizaciones, escalas de puntuación, sujetos y modos de administración (cf. Collados Aís et al. 2011). El parámetro entonación, conjuntamente con los parámetros acento y agradabilidad de la voz (Cheung 2013; Iglesias Fernández 2007, 2011; Stévaux 2007, 2011), independientemente del formato de cuestionario, ha obtenido de forma consistente resultados que denotan la relativa poca importancia que los usuarios le otorgarían a priori frente al resto de los parámetros de calidad. No obstante, el hecho de que se incluyesen los distintos parámetros en el mismo cuestionario y que, de alguna manera, fuesen 'enfrentados' entre sí, podría ser uno de los motivos por los que a los parámetros no verbales se les suela atribuir menor importancia que a los verbales. Por otra parte, la propuesta de un listado conjunto que englobaba todos los parámetros implicados en la calidad de la interpretación dificultaba un examen más exhaustivo de cada uno de ellos de forma individual, de manera que permitiese analizar pormenorizadamente las razones que los sujetos pudieran tener para asignarles un determinado valor a la hora de evaluar una interpretación.

A fin de comprobar si efectivamente el hecho de que la entonación apareciese en el mismo cuestionario junto a otros parámetros señalados como 'más importantes', como transmisión correcta o cohesión, le pudiese restar un protagonismo que sí se ha puesto de manifiesto en los estudios de evaluación (Collados Aís 1998; Garzone 
2003; Holub 2010), se presenta esta investigación que indaga única y exclusivamente los distintos aspectos de la entonación del intérprete y que parte de los intraparámetros propios (Pradas Macías 2006) de este criterio de calidad. De esta forma, mediante este nuevo planteamiento, se pretende que el usuario encuestado no se sienta «intimidado» por otros parámetros que, teóricamente, son más importantes para la calidad de una IS, otorgando, ya de forma no consciente, un menor valor a la entonación.

A continuación, tras exponer brevemente la investigación sobre la entonación del intérprete y sobre las expectativas y la evaluación que se ha llevado a cabo hasta la fecha en el ámbito de la calidad de la IS, presentaremos el trabajo realizado, así como las conclusiones generales que se pueden extraer de los resultados del mismo, en combinación con otros estudios que han analizado la entonación del intérprete.

\section{Estado de la cuestión ${ }^{1}$}

\subsection{La entonación del intérprete}

Desde los inicios de los estudios de la interpretación se ha venido destacando la importancia que reviste una buena presentación para la calidad de una interpretación. En este sentido, se ha resaltado la trascendencia de la voz del intérprete, que no ha de ser ni desagradable ni monótona (Katz 1989), debiendo este profesional hacer uso de los medios prosódicos que tiene a su alcance (Déjean Le Féal 1981: 94). La razón por la que una voz agradable casi siempre llevaría varios puntos de ventaja (Herbert 1970) podría radicar, entre otras razones, en que ayudaría a convencer al oyente de la calidad de la idea formulada (Gile 1991). En el ámbito de las asociaciones profesionales, tampoco se ha obviado la relevancia de aspectos tales como la voz o la entonación (véase, entre otros, AIIC 2004). En un estudio temprano llevado a cabo por la AIIC (1986 apud Mazzetti 2005: 127) ya se reflejaba el peso de la entonación y del lenguaje corporal sobre la comprensión en la comunicación oral. A pesar de ello, los aspectos vocales no verbales empezaron a estudiarse después de que ya se hubiese iniciado la investigación sobre los aspectos verbales. La justificación puede estar en el propio desarrollo tardío de estudios específicos y de técnicas instrumentales adecuados para la medición de este tipo de parámetros, ya que incluso en el ámbito de la psicología y la lingüística, la fonética o la psicofonética, los primeros estudios se centraron en los aspectos verbales.

En cuanto a los trabajos vinculados directamente con el tema que nos ocupa se encuentra el realizado por Daró en 1990, que estudia las diferencias de tono (Fo) entre distintas lenguas de trabajo de una intérprete de conferencias. La autora concluye que las diferencias son mayores cuando la interpretación se realiza hacia la lengua no materna por la mayor tensión que eso implica. En la misma línea se sitúa Schweda Nicholson (1992: 97), que justifica la interpretación hacia la lengua materna precisamente debido al mayor automatismo en la producción de los rasgos paralingüísticos y prosódicos:

An additional argument put forth in support of interpreters working solely into an 'A' language is that the production of correct prosodic (intonation and stress) patterns does not require the conscious attention of a native speaker. As well, one can claim that certain paralinguistic features (i.e., tone and loudness of voice, rhythm, and tempo) are not always given conscious attention as we speak. 
En 1994, Shlesinger publica un trabajo que nuevamente tiene a la voz como objeto de estudio en la investigación, concretamente su influencia sobre el nivel de retención de una IS. Su trabajo muestra que la retención a partir de una IS es inferior al grado de retención que se alcanza a partir de textos leídos. La autora comparó la emisión vocal de la IS con la de la lectura, concluyendo que existirían rasgos peculiares propios de la entonación del intérprete y, en general, de su emisión vocal. Estas características se centran fundamentalmente en un incremento importante de pausas no gramaticales en posiciones «no usuales» y en una prosodia específica con acentuación de elementos que en el habla espontánea o leída no se producirían.

En 2001, Collados Aís publica un trabajo que intenta averiguar si la monotonía en la voz del intérprete afecta la tasa de retención de la información transmitida a través de la IS. Para ello, compara dos IS: una con una entonación monótona y otra con una entonación no monótona. Los resultados muestran una tendencia a una mayor retención por parte de los usuarios especializados en la IS no monótona que en la IS monótona, aunque sin llegar a la significación estadística. Estos resultados estarían en consonancia con los obtenidos por Holub (2010) respecto al nivel de comprensión que se alcanzaría en una interpretación monótona y en otra no monótona. Asimismo, se pondrían de manifiesto los efectos de la monotonía en la interpretación, en el sentido que ya se ha expuesto en trabajos realizados en el ámbito de la lingüística y que inciden en los efectos de los elementos suprasegmentales sobre el nivel semántico pragmático (cf. Barranco-Droege, Collados Aís y Pazos Bretaña 2011; Jiménez Ivars 2011, Llisterri 2014).

Ahrens (2005) aporta un estudio exhaustivo sobre la entonación estudiada a través de un corpus de IS inglés-alemán. Sus conclusiones apuntan también a aspectos específicos del estilo discursivo del intérprete condicionados por el propio proceso de la IS y las condiciones comunicativas específicas en las que se produce (Ahrens 2005: 230). El intérprete tiende a una segmentación prosódica mayor del texto por motivos estratégicos de segmentación de contenido (Ahrens 2005: 227). Por su parte, Nafá (2005), en una amplia investigación dedicada íntegramente a la entonación a partir de un corpus extraído del Parlamento Europeo, muestra el uso de estrategias entonacionales y retóricas a las que recurren los intérpretes para la comunicación en su ejercicio profesional, y que se centran en la organización y estructuración de sus discursos. Estas estrategias no se aplican en todos los casos ni durante toda una interpretación, posiblemente porque esta entonación comunicativamente aceptable se ve alterada por las demandas cognitivas del proceso de la IS (Nafá 2005: 678).

\subsection{La entonación en los estudios de expectativas y evaluación de la calidad de la interpretación}

Los estudios de expectativas de calidad de la interpretación en distintos grupos de usuarios -provenientes de distintos ámbitos o especialidades y con independencia de otros factores como el género o la edad-, así como en los propios intérpretes, han venido mostrando una incidencia claramente menor de los parámetros no verbales frente a los parámetros verbales, tales como la transmisión correcta y la cohesión (cf. Kurz 2001, 2003; Collados Aís et al. 2007; Collados Aís et al. 2011). Únicamente en trabajos en los que el foro en el que tienen lugar - cine y televisión- determinan unas características específicas de los sujetos (Kurz \& Pöchhacker 1995; Russo 2005), los 
parámetros no verbales adquieren una posición algo más relevante que en los anteriores, aunque sin modificar el ranking habitual de preferencias. Sin embargo, en un experimento sobre evaluación de la calidad realizado por Collados Aís en 1998 no se confirmaron estas expectativas en cuanto a la entonación monótona de la IS. Por el contrario, se constató una incidencia alta de la entonación monótona no sólo sobre la evaluación global de trabajo del intérprete, sino también sobre los demás parámetros de calidad -verbales y no verbales- barajados en la investigación y sobre aspectos tales como la fiabilidad o la profesionalidad del intérprete. Estos resultados denotarían la influencia, incluso no consciente, de los elementos vocales no verbales sobre los juicios emitidos por los oyentes sobre el propio intérprete, su personalidad y credibilidad, y refrendarían en interpretación los datos de las investigaciones llevadas a cabo en el ámbito de la psicología (cf. Collados Aís 1998); algo que se ha confirmado en experimentos posteriores sobre otros aspectos no verbales como las primeras impresiones que se forman los sujetos sobre el intérprete de simultánea (cf. García Becerra 2012).

Si bien, en el caso concreto de la entonación, la replicación posterior del experimento no corroboró estos resultados (Collados Aís 2007), aun tratándose de sujetos muy similares, estudios anteriores (Garzone 2003) y posteriores (Holub 2010) sí ratificaron esta incidencia. Se comprobó, además, que la influencia de la entonación monótona sobre la evaluación sería también diferente según el tipo de usuario, a pesar de que la hipótesis de partida no preveía estos resultados dado que se consideraba que su influencia debía de ser menor que en el caso de las expectativas. De esta forma se comprobó que, por ejemplo, los sujetos provenientes del ámbito de ciencias serían menos sensibles a las características de la entonación del intérprete que los de humanidades, no solo en sus expectativas, sino también en su evaluación de una interpretación concreta (Collados Aís 2010). Asimismo, el orden de escucha de una interpretación con entonación monótona frente a una no monótona parece producir diferencias importantes en la evaluación, más evidentes en las franjas en las que tenía lugar la valoración que en las propias diferencias de puntuaciones obtenidas por las interpretaciones (Collados Aís 2008). Otros estudios realizados siguiendo metodologías provenientes de las ciencias sociales y con un carácter más cualitativo (grupos de discusión) indican que los usuarios partirían efectivamente de un cierto grado de monotonía del intérprete en su modelo o estereotipo de interpretación (Collados Aís 2009), lo que en todo caso podría matizar los resultados de los distintos trabajos efectuados sobre la entonación del intérprete y subrayaría la necesidad de partir de este modelo para interpretar los posibles resultados que se obtengan en cualquier estudio sobre el tema, puesto que la entonación no monótona se consideraría un elemento diferenciador, y se valoraría positivamente que un intérprete 'rompiese' con ese modelo previo que incluiría cierta monotonía en la entonación.

\section{Estudio empírico}

\subsection{Antecedentes}

Este estudio tiene su antecedente inmediato en una investigación realizada por las integrantes del grupo de investigación ECIS (Evaluación de la Calidad en IS) ${ }^{2}$ sobre expectativas y evaluación de la calidad en IS y que incluía el estudio piloto de la 
investigación que ahora presentamos (cf. Collados Aís et al. 2007). En ese trabajo inicial, los usuarios - profesores de la Facultad de Derecho de la Universidad de Las Palmas de Gran Canaria- contestaron a un cuestionario que indagaba sobre la conceptualización de los distintos parámetros de calidad que tradicionalmente se han considerado en la investigación sobre este tema, con la particularidad de que cada grupo de sujetos respondía a las preguntas sobre un parámetro de calidad específico. En concreto, para el parámetro entonación, los resultados indicaron que los usuarios eran buenos conocedores de los intraparámetros normalmente asociados a la entonación en la bibliografía interdisciplinar. De hecho, incluían referencias a la entonación tanto desde un punto de vista lingüístico, vinculándola a aspectos tales como la modulación o el énfasis, como desde un punto de vista psicológico, haciendo alusión a las implicaciones emocionales (aburrimiento o pérdida de atención) y a los efectos directos de una entonación monótona. La realización de este estudio piloto reveló también la posible conexión entre la entonación -parámetro vocal no verbal- y determinados parámetros de contenido, especialmente la cohesión y la transmisión correcta (Collados Aís 2007). Este vínculo pondría de relieve la gran importancia de la entonación para el acceso al mensaje por parte de los receptores debido a que podría determinar la comprensión del discurso.

\subsection{Objetivos e hipótesis}

El objetivo principal de esta investigación se centra en el análisis de las especificidades del parámetro de calidad entonación como un elemento importante que puede afectar a la evaluación de la calidad de una IS. Se espera que las respuestas de los sujetos contribuyan a dilucidar aspectos que hasta ahora han pasado desapercibidos, teniendo en cuenta que este parámetro se viene estudiando simultáneamente con otros parámetros sobre los que existe práctica unanimidad acerca del peso que adquieren en la evaluación de la calidad de una interpretación, tales como la transmisión correcta.

Las hipótesis de las que partimos son dos: (1) el parámetro entonación puede ser considerado como un parámetro que afecta notablemente la evaluación de una IS; (2) el estudio pormenorizado de las conceptualizaciones de las que parte el usuario -incluidos los efectos que previsiblemente podría tener sobre su comprensión y evaluación de una IS- podrá poner de manifiesto vías que apoyen nuestra primera hipótesis.

\subsection{Metodología}

\subsubsection{Sujetos}

En total respondieron al cuestionario 15 sujetos, todos ellos profesores de la Universidad de Jaén. En cuanto al área del conocimiento al que pertenecen, cuatro provienen del ámbito de la ciencia y tecnología, seis sujetos de las ciencias sociales, cuatro de la rama biosanitaria y un sujeto del ámbito de las humanidades. De los 15 sujetos, siete son mujeres $(46,66 \%)$ y ocho hombres $(53,33 \%)$. En cuanto a la edad, un $73,33 \%$ se sitúa en la franja de los 30 a 45 años, y el resto se distribuye entre las restantes franjas (menor de 30 años, entre 46 y 60 años y mayor de 60 años). 


\subsubsection{Material y método}

El cuestionario sobre el parámetro entonación se diseñó a partir del cuestionario base que elaboraron los miembros del grupo ECIS para todos los parámetros y que se basó en el utilizado para el estudio piloto (cf. Collados Aís 2007) (véase nota 3), si bien se completó y amplió en función de las características concretas del parámetro entonación. Se realizó una encuesta presencial con la intención de alcanzar una mayor tasa de respuesta, teniendo en cuenta que, al tratarse de una investigación sobre parámetros, el número de cuestionarios que se necesitaba era bastante elevado. De esta forma, algunas investigadoras del grupo ECIS se desplazaron a la Universidad de Jaén durante varios días hasta recabar el número suficiente de cuestionarios cumplimentados para cada uno de los parámetros de calidad investigados. ${ }^{3}$

Aunque se incluyeron un total de once preguntas, en este trabajo nos referiremos únicamente a las seis preguntas cerradas. Para la preparación del cuestionario se recurrió a la bibliografía sobre la materia, especialmente en lo que se refiere a las preguntas que proponen los intraparámetros asociados con mayor frecuencia a la entonación y a los efectos más relatados en torno a una entonación monótona. De igual manera, se utilizaron también como base los resultados del estudio piloto (Collados Aís 2007: 167-169).

En concreto, las preguntas formuladas en el cuestionario que analizaremos a lo largo de este estudio, fueron las siguientes:

1. En su opinión, ¿es frecuente la entonación monótona en una interpretación simultánea (5: siempre - 1 nunca)?

2. Cuando escucha una interpretación simultánea monótona, ¿cuál o cuáles de los siguientes elementos o intraparámetros de la entonación le molestan más (5: máxima molestia - 1: mínima molestia)? El tono plano, la falta de modulación, la velocidad demasiado lenta, el volumen plano y sin oscilaciones, la falta de diferenciación entre las frases, la acentuación inadecuada, otros (especifique por favor).

3. ¿Considera Vd. que una emisión monótona le puede afectar negativamente también en la valoración de otros parámetros de calidad de una interpretación simultánea? Especifique por favor el efecto sobre dicha valoración (5: máximo efecto - 1 : mínimo efecto): Acento, agradabilidad de la voz, cohesión lógica, fluidez, dicción, estilo, gramaticalidad, terminología, transmisión correcta, transmisión completa, fiabilidad del intérprete, profesionalidad del intérprete, otros (especifique por favor)

4. ¿Puede decirnos qué tipo de efecto o efectos le causa la emisión monótona como oyente de una interpretación simultánea (5: máximo efecto - 1: mínimo efecto)? Distrae mi atención, me desconcentra, me aburre, dificulta mi comprensión, me pone nervioso/a, me cansa, hace que tenga que esforzarme más, otros (especifique por favor)

5. ¿Le influye la emisión monótona cuando evalúa negativamente una interpretación simultánea (5: máxima influencia - 1: mínima influencia)?

6. ¿Le influye la entonación no monótona cuando evalúa positivamente una interpretación simultánea (5: máxima influencia - 1: mínima influencia)?

\subsection{Resultados y Discusión}

En la exposición y discusión de los resultados seguiremos el orden marcado por el propio cuestionario. 


\subsubsection{Frecuencia}

\section{A. Resultados}

A esta pregunta contestan 14 de los 15 sujetos de la muestra, quienes situaron la frecuencia de la entonación monótona en un grado de aparición medio/alto con una media de 3,5 sobre 5 (véase tabla 1). La desviación típica puede calificarse de baja (.760).

TABLA 1

Frecuencia: media y desviación típica

\begin{tabular}{|l|c|c|c|c|c|}
\hline & $\mathrm{N}$ & Mínimo & Máximo & Media & Desv. típica \\
\hline Frecuencia & 14 & 2 & 5 & 3,50 &, 760 \\
\hline
\end{tabular}

En cuanto a los porcentajes en la distribución de las respuestas (véase tabla 2), un 7,1\% responde asigna un 2 a la frecuencia de aparición, un 42,9\%, un 3, un 42,9\%, un 4 , y un $7,1 \%$, un 5 . Ningún sujeto considera que la frecuencia de la entonación monótona en IS sea nula (1).

TABLA 2

Frecuencia: distribución respuestas

\begin{tabular}{|ll|c|c|c|}
\hline & & Frecuencia & $\%$ & \% válido \\
\hline Válidos & 2 & 1 & 6,7 & 7,1 \\
& 3 & 6 & 40,0 & 42,9 \\
& 4 & 6 & 40,0 & 42,9 \\
& 5 & 1 & 6,7 & 7,1 \\
& Total & 14 & 93,3 & 100,0 \\
& Perdidos & 1 & 6,7 & \\
Total & & 15 & 100,0 & \\
\hline
\end{tabular}

\section{B. Discusión}

La lectura de los datos permite inferir que los sujetos consideran, en su gran mayoría, que las interpretaciones simultáneas que pueden ser calificadas de monótonas tienen una frecuencia de aparición media o alta $(92,9 \%)$. Nos parecen muy indicativos los porcentajes, ya que la mitad de los encuestados responden que la entonación monótona es muy frecuente o aparece siempre en una IS. Además, ningún sujeto ha marcado el valor 1, lo que indicaría una nula aparición, y solamente un sujeto ha marcado el valor 2 (poca frecuencia). Por otra parte, el hecho de que la desviación típica sea de .760 confirmaría que existe una gran coincidencia entre los sujetos a la hora de calificar el grado de frecuencia de aparición de la entonación monótona.

Estos resultados estarían en concordancia con los obtenidos por el grupo de discusión 'expertos' (profesoras de interpretación, psicología y lingüística) del trabajo de Collados Aís (2008), en el que varios de los sujetos calificaron como bajas las valoraciones que habían otorgado a la entonación de los intérpretes durante la evaluación que realizaron de su trabajo. Asimismo, corroboraría los resultados obtenidos en el grupo de discusión 'usuarios' del trabajo de Collados Aís (2009), en el que los sujetos (profesores de ciencia política de la Universidad de Granada) asumían, e incluso justificaban, una cierta monotonía por parte de los intérpretes, además de 
constatar la 'entonación sui generis' de estos profesionales. El hecho de que, en la práctica, la frecuencia de aparición de una cierta monotonía en el intérprete pueda ser considerada como media/alta podría explicar por qué los usuarios justifican su presencia en la interpretación, dado que el modelo de IS o el estereotipo de intérprete del que parten incluiría efectivamente esa cierta entonación monótona, al igual que otros aspectos que la definirían como propia de la IS, es decir, como entonación sui generis (Collados Aís 2008).

\subsubsection{Intraparámetros}

\section{A. Resultados}

A esta pregunta responden los 15 sujetos (véase tabla 3). Todos marcan los intraparámetros «tono plano» $\mathrm{y}$ «falta de diferenciación entre las frases» como molestos. A estos factores les siguen, en orden de número de menciones, una «acentuación inadecuada» y una "velocidad demasiado lenta» (14 de los 15 sujetos en cada caso), y la «falta de modulación» y el «volumen plano y sin oscilaciones» (13 sujetos). En cuanto a la media resultante, la «falta de diferenciación entre las frases» se sitúa con un 3,67 como el elemento más molesto, seguido de la «falta de modulación» y del «tono plano». Todos estos intraparámetros obtienen medias por encima de $3(3,67,3,31$ y 3,13 , respectivamente). El intraparámetro al que menos importancia se le otorgaría en cuanto a la molestia producida sería el de la «velocidad demasiado lenta». Si consideramos la desviación típica de todos los factores, observamos que los valores más bajos se producen en la «falta de modulación», en la «velocidad demasiado lenta»y en el «volumen plano y sin oscilaciones» $(.751, .832, .864)$. La mayor desviación típica se registra en la «falta de diferenciación entre las frases» (1.397).

TABLA 3

Intraparámetros

\begin{tabular}{|l|c|c|c|c|c|}
\hline & $\mathrm{N}$ & Mínimo & Máximo & Media & Desv. típica \\
\hline Tono plano & 15 & 1 & 5 & 3,13 & 1,060 \\
Falta de modulación & 13 & 2 & 4 & 3,31 &, 751 \\
Velocidad demasiado lenta & 14 & 1 & 4 & 1,86 &, 864 \\
Volumen plano y sin oscilaciones & 13 & 1 & 4 & 2,77 &, 832 \\
Falta de diferenciación entre las frases & 15 & 1 & 5 & 3,67 & 1,397 \\
Acentuación inadecuada & 14 & 1 & 5 & 2,43 & 1,284 \\
\hline
\end{tabular}

\section{GRÁFICO 1}

\section{Media intraparámetros}

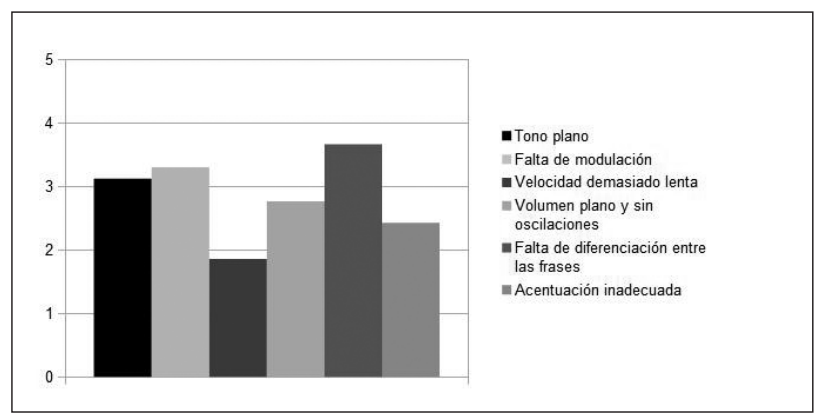




\section{B. Discusión}

El análisis de los resultados permite vislumbrar dos vías de conceptualización. Por una parte, el hecho de que haya dos intraparámetros que marquen todos los sujetos y que, en general, todos los intraparámetros sean referenciados por la mayoría de los sujetos de la muestra (al menos 13) parece indicar que todos tienen una conceptualización común del parámetro entonación. Desde nuestro punto de vista, este gran número de menciones a todos los intraparámetros permite deducir que han sido reconocidos y compartidos como tales por los sujetos. Por otra parte, y entrando en los resultados concretos, la «falta de diferenciación entre las frases» sería el ítem que más molestaría a los participantes. Así, se pone de manifiesto un vínculo entre la mayor molestia que puede provocar una entonación monótona para estos sujetos y los parámetros de contenido que tradicionalmente se ha presentado como esenciales en los estudios de expectativas (i.e. cohesión y la transmisión correcta del sentido). Por el contrario, el intraparámetro menos molesto sería la «velocidad demasiado lenta», lo que, en principio, no afectaría de manera tan directa el nivel de comprensión.

Estos resultados son coherentes con los obtenidos en el estudio piloto (cf. Collados Aís 2007) y estarían en consonancia con la relación establecida entre entonación y procesamiento de la estructura discursiva por parte del oyente, que sería facilitada precisamente por la función cohesiva e integradora de la entonación (cf. Barranco-Droege, Collados Aís y Pazos Bretaña 2011). En este sentido, es clara la asociación que se establece entre la entonación, como parámetro de calidad no verbal, y la cohesión, como parámetro verbal, y por ende también con la transmisión correcta.

\subsubsection{Interacciones}

\section{A. Resultados}

Todos los sujetos consideran que la entonación monótona podría afectar los parámetros de calidad voz, cohesión, fluidez, terminología y transmisión correcta y las variables fiabilidad y profesionalidad. Catorce señalan que la monotonía también incidiría en la percepción del resto de parámetros (véase tabla 4). En cuanto a la media del posible efecto de la entonación monótona sobre estos elementos, sobresale el parámetro cohesión con un 3,60 de influencia, seguido de la transmisión correcta con un 3,53 y la profesionalidad con un 3,27. Todos los demás parámetros han obtenido una media inferior a 3, salvo fluidez (3). En lo que respecta a la desviación típica, el valor más bajo se registra en cohesión con un .910 que, junto con dicción, son los únicos parámetros que obtienen una desviación típica por debajo de 1 .

TABLA 4

Interacciones

\begin{tabular}{|l|c|c|c|c|c|}
\hline & $\mathrm{N}$ & Mínimo & Máximo & Media & Desv. típica \\
\hline acento & 14 & 1 & 5 & 2,50 & 1,401 \\
voz & 15 & 1 & 5 & 2,73 & 1,033 \\
cohesión & 15 & 2 & 5 & 3,60 &, 910 \\
dicción & 14 & 1 & 4 & 2,71 &, 944 \\
estilo & 14 & 1 & 4 & 2,79 & 1,251
\end{tabular}




\begin{tabular}{|l|l|l|l|l|l|} 
fluidez & 15 & 1 & 5 & 3,00 & 1,254 \\
gramaticalidad & 14 & 1 & 5 & 2,43 & 1,158 \\
terminología & 15 & 1 & 5 & 2,67 & 1,234 \\
t. correcta & 15 & 1 & 5 & 3,53 & 1,125 \\
t. complata & 14 & 1 & 5 & 3,07 & 1,207 \\
fiabilidad & 15 & 1 & 5 & 3,00 & 1,069 \\
profesionalidad & 15 & 1 & 5 & 3,27 & 1,387 \\
\hline
\end{tabular}

\section{B. Discusión}

Resulta llamativa la coincidencia y coherencia de estos resultados con los obtenidos en la pregunta anterior respecto a los posibles elementos más molestos de una entonación monótona. Si allí sobresalía la «falta de diferenciación entre las frases»aspecto muy vinculado a la cohesión-, en esta pregunta el parámetro que, en opinión de los sujetos, resultaría más afectado por la monotonía del intérprete sería precisamente la cohesión. No es de extrañar, por tanto, que el siguiente parámetro más afectado por la entonación monótona sea la transmisión correcta, seguido de la profesionalidad del intérprete. Estos datos parecen indicar que el usuario es muy consciente de los problemas que puede conllevar la monotonía sobre la propia comprensión del mensaje, más allá de la mera molestia. Además, reforzarían los resultados de estudios anteriores que han tenido por objeto la comprensión o la retención de información como efectos de una interpretación monótona frente a una interpretación no monótona (Collados Aís 2001; Holub 2010).

\subsubsection{Efectos}

\section{A. Resultados}

Todos los sujetos señalan como efectos de la entonación monótona «me distrae» y «me desconcentra», 14 sujetos marcan también «dificulta mi comprensión» y «hace que tenga que esforzarme más», y 13 indican los tres restantes (véase tabla 5). Si nos atenemos a la media, observamos que los efectos más importantes serían el aburrimiento $(3,92)$, el cansancio $(3,69)$ y el esfuerzo añadido que la entonación monótona requeriría por parte de los sujetos $(3,50)$. Como efectos menos importantes se consideraría que ponga nervioso al oyente $(1,92)$ y que dificulte la comprensión, aunque esta última con una puntuación bastante más alta $(2,64)$. La mayor coincidencia entre los sujetos se produce en los efectos «aburrimiento» $\mathrm{y}$ «nerviosismo», con desviaciones típicas idénticas (.954) y la menor coincidencia se observa en la opinión acerca del mayor esfuerzo que les requeriría la entonación monótona del intérprete (1.606).

TABLA 5

Efectos

\begin{tabular}{|l|c|c|c|c|c|}
\hline & $\mathrm{N}$ & Mínimo & Máximo & Media & Desv. típica \\
\hline distrae & 15 & 1 & 5 & 3,07 & 1,280 \\
desconcentra & 15 & 1 & 5 & 3,00 & 1,309 \\
aburre & 13 & 2 & 5 & 3,92 &, 954 \\
dificulta & 14 & 1 & 5 & 2,64 & 1,393 \\
(pone) nervioso & 13 & 1 & 4 & 1,92 &, 954 \\
cansa & 13 & 1 & 5 & 3,69 & 1,182 \\
(requiere mayor) esfuerzo & 14 & 1 & 5 & 3,50 & 1,605 \\
\hline
\end{tabular}


GRÁFICO 2

Media efectos

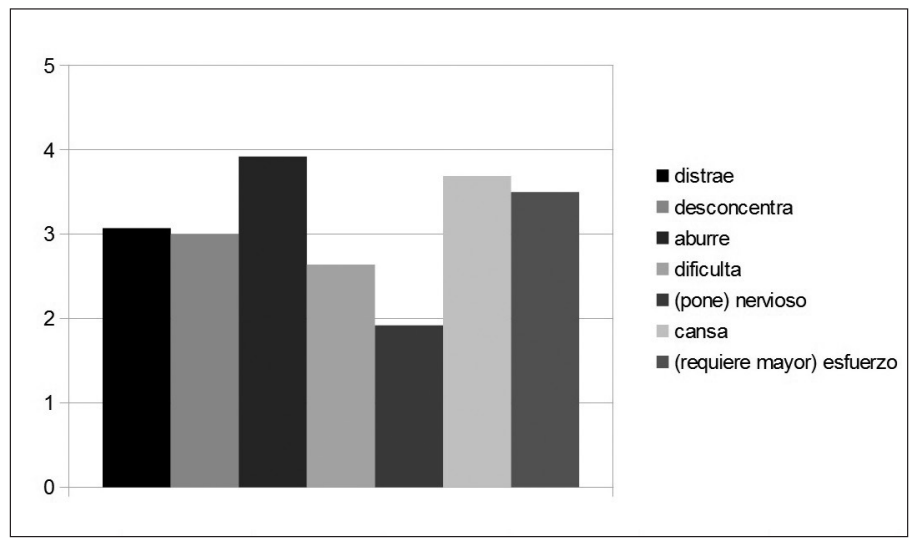

\section{B. Discusión}

Podemos deducir de las respuestas anteriores que, si nos atenemos al número de sujetos que marcan cada uno de los elementos, los aspectos citados en el cuestionario como posibles efectos de una entonación monótona parecen haber sido bastante certeros, puesto que ninguno ha obtenido menos de 13 menciones. En cuanto a los resultados, solamente dos efectos no alcanzan un 3 de media: el nerviosismo que puede producir y las dificultades añadidas respecto a la comprensión. Estos datos parecen indicar que muy posiblemente el primer efecto y el más perceptible es efectivamente el aburrimiento, mientras que los efectos sobre la comprensión, que sugieren las respuestas de las preguntas anteriores, aparecen en un segundo lugar, al menos desde un punto de vista temporal.

Un dato interesante es que la mayor coincidencia entre los sujetos se produce precisamente en los efectos más y menos señalados: el aburrimiento y el nerviosismo. De hecho, son los dos únicos elementos cuya desviación típica no supera el 1 (.954 en ambos). En todo caso, los efectos que se producen en el oyente se evidencian, tal y como ha sido estudiado en el ámbito de la psicología, en emociones situadas en estados de ánimo de los polos negativos/pasivos (cf. Collados Aís, 1998, BarrancoDroege, Collados Aís y Pazos Bretaña 2011).

\subsubsection{Influencia negativa}

\section{A. Resultados}

Todos los sujetos afirman que la entonación monótona incide de alguna manera en la evaluación de la IS (véase tabla 6). Ningún sujeto opina que tenga una influencia mínima cuando evalúa una IS, aunque un 13,33\% la califica de escasa (2). En la parte media de la escala de influencia se sitúa un $40 \%$ de las respuestas. El resto, es decir, un $46,66 \%$, estima que dicha influencia es alta $(33,33 \%)$ o máxima $(13,33 \%)$. En cuanto a la media general, el grado de influencia asciende a 3,47, es decir, por encima de una influencia media, con una desviación típica que no supera el 1 (.915). 
TABLA 6

Influencia negativa

\begin{tabular}{|l|c|c|c|c|c|}
\hline & $\mathrm{N}$ & Mínimo & Máximo & Media & Desv. típica \\
\hline Influye negativamente & 15 & 2 & 5 & 3,47 &, 915 \\
\hline
\end{tabular}

\section{B. Discusión}

Estos resultados denotan que los sujetos son conscientes de la influencia negativa de una entonación monótona sobre la valoración de una IS, aunque la coincidencia al respecto es algo menor que en otros apartados. Sin embargo, los datos no se corresponden con los que se suelen obtener en cuestionarios de expectativas que indagan en general sobre todos los parámetros de calidad que pueden influir en la evaluación de la calidad de una IS. De hecho, ningún usuario ha valorado dicho efecto con un 1 (mínima influencia). Da la impresión de que si se enfrenta a los usuarios a parámetros aislados, adquieren mayor conciencia sobre la importancia de los mismos. Por el contrario, los resultados sí parecen más coherentes con los obtenidos en trabajos anteriores sobre evaluación de la interpretación, en los que también se les ha expuesto a parámetros concretos. Como dato adicional, sería interesante relacionar estos resultados con los obtenidos por los grupos de discusión (Collados Aís 2008, 2009), dado que los participantes de esos trabajos, a pesar del reconocimiento de los efectos negativos de la entonación sobre la calidad de la interpretación, sí parecían justificarla debido a la dificultad que entraña el proceso de la IS y los consiguientes efectos intrínsecos que pueden derivar en una entonación sui generis, caracterizada por cierta monotonía.

\subsubsection{Influencia positiva}

\section{A. Resultados}

Todos los sujetos indican que la entonación no monótona influye en la evaluación positiva de una IS (véase tabla 7). Ningún sujeto considera que tenga poco o ningún efecto sobre la valoración. De hecho, un 33,33\% señala que la influencia es media, y un $66,67 \%$ se refiere a esa influencia como alta (40\%) o máxima $(26,67 \%)$. La media resultante sería de un 3,93 y la desviación típica, de un .799.

TABLA 7

Influencia positiva

\begin{tabular}{|l|c|c|c|c|c|}
\hline & $\mathrm{N}$ & Mínimo & Máximo & Media & Desv. típica \\
\hline Influye positivamente & 15 & 3 & 5 & 3,93 &, 799 \\
\hline
\end{tabular}

\section{B. Discusión}

La interpretación de los resultados permite inferir que la entonación no monótona del intérprete adquiere un papel relevante para que estos sujetos evalúen positivamente una IS. Lo cierto es que el hecho de que ningún sujeto señale que esa influencia es mínima o baja resulta bastante indicativo. De igual manera, el valor de la desviación típica es bajo, lo que denota una gran coincidencia entre los sujetos a la hora de determinar la incidencia de la entonación no monótona en una valoración positiva de la IS. Al igual que en el apartado anterior, los datos parecen sugerir una 
conciencia bastante alta respecto a la influencia real de la entonación sobre la evaluación de una interpretación. No obstante, creemos que una de las aportaciones más interesantes de este estudio es el establecimiento de un vínculo de los resultados con el modelo previo de la entonación de un intérprete con el que cuentan los usuarios. De esta forma, los efectos negativos podrían considerarse menores que los positivos dado que el modelo previo incluiría una cierta monotonía. Esta interpretación estaría en consonancia con lo dicho por una de las participantes en el grupo de discusión 'expertos' del estudio de Collados Aís (2008) cuando afirmaba que «una buena interpretación sería precisamente aquella que rompe con su propio modelo», es decir que haga uso de una entonación diferente a la que normalmente se le atribuye a un intérprete, algo que el usuario premiaría.

\section{Conclusiones}

Los resultados obtenidos en la presente investigación no coinciden con los resultados cuantitativos de los estudios sobre expectativas generales de calidad de una IS por parte de los usuarios (Collados Aís et al. 2011). A pesar de que esta investigación también puede considerarse un estudio de expectativas, los resultados se situarían más en la línea de los trabajos de evaluación de una IS (Collados Aís 1998, Holub 2010), ya que la habitual preeminencia de los parámetros verbales sobre los no verbales no parece confirmarse en los datos de esta muestra.

Si en la investigación tradicional sobre expectativas, la entonación ha venido ocupando un lugar muy alejado de los primeros puestos del ranking de preferencias - copado por la transmisión correcta y la cohesión-, situándose en la parte más baja de la tabla junto a los parámetros agradabilidad de la voz y acento (Iglesias Fernández 2011; Stévaux 2011), en el presente estudio los usuarios resaltan su importancia y reconocen su influencia en una hipotética evaluación de una IS, si bien esta influencia se situaría más en el polo positivo que en el negativo, es decir, que se trataría más bien de una influencia positiva sobre su valoración de una IS, que de una influencia negativa. Esto podría estar estrechamente relacionado con la frecuencia de aparición de la entonación monótona en IS, que puede ser considerada alta, y refrendaría los resultados de investigaciones anteriores más cualitativas que estudian los modelos de evaluación que los usuarios realizan (Collados Aís 2008, 2009) y que apuntan a un modelo de IS que incluye la entonación monótona y sui generis del intérprete (Shlesinger 1994, Ahrens 2005). En todo caso, además de los efectos psicológicos que produciría una interpretación con entonación monótona en los oyentes, como efectos más evidentes, se añadiría su influencia, a través de los elementos suprasegmentales (Llisterri 2014), sobre la comprensión y la atención al escuchar una IS, dificultando la primera y mermando la segunda. Así, el parámetro entonación parece traspasar el ámbito no verbal, entendido como un ámbito importante para la forma, y se convierte en un criterio que también afectaría al contenido.

El hecho de que metodológicamente este estudio analice la entonación sin referencia directa al orden de importancia del resto de parámetros parece que efectivamente la coloca en un lugar bien distinto del ranking habitual de importancia. Vendría, por tanto, a corroborar la necesidad de los experimentos que se acercan a la evaluación real de los usuarios, completando dichas investigaciones con explicaciones plausibles en cuanto a la dicotomía reflejada entre los estudios de expectativas 
y los de evaluación. Los usuarios diferencian claramente entre dos conceptualizaciones básicas de la entonación, tal y como ya se puso de manifiesto en el estudio piloto que precede a esta investigación (Collados Aís 2007). Por una parte, aquella que se fundamenta en aspectos psicológicos tales como las inferencias sobre actitudes o estados de ánimo del intérprete, o incluso sobre rasgos permanentes; $y$, por otra parte, la que se basa en los aspectos más lingüísticos y cuyo ítem fundamental sería la comprensión.

De este modo, se ratificarían los resultados de estudios realizados en el ámbito de la lingüística respecto a la función cohesiva de la entonación, así como en el de la psicología -el que más se ha tenido en cuenta hasta ahora- respecto a los efectos sobre el oyente (Barranco-Droege, Collados Aís y Pazos Bretaña 2011). Asimismo se explicarían los resultados de estudios realizados en el ámbito de la interpretación sobre la tasa de retención de información y el nivel de comprensión según el tipo de entonación (Collados Aís 2001, Holub 2010). Concretamente, la explicación parece estar relacionada con los efectos que la entonación monótona podría tener sobre el parámetro cohesión y, por ende, a través de esta vía, sobre la transmisión correcta, dificultando o incluso evitando el acceso al mensaje de la interpretación. Las implicaciones de estos datos son claras si tenemos en cuenta que la transmisión correcta es el parámetro de calidad por excelencia ya que afecta al sentido mismo de la interpretación, su función principal.

Desde el punto de vista de los efectos emocionales, se parte de una incidencia notable sobre el propio usuario en cuanto a su actitud frente a la interpretación. Si la entonación monótona produce aburrimiento en el oyente como efecto más destacado (véase apartado 3.4.4.), y si correlaciona fundamentalmente con el parámetro cohesión y con el parámetro transmisión correcta (véase apartado 3.4.3.), pasaría de ser un mero componente formal que podría realzar el éxito de una interpretación como componente vocal no verbal, a adquirir también un papel como componente vocal verbal en el fondo. La trascendencia teórica y práctica de esta conclusión es, a nuestro modo de ver, considerable ya que permitiría situar en igualdad de importancia a la entonación, como parámetro vocal no verbal, y a los parámetros de contenido en la interpretación (véase apartado 2.1.), algo que debería tenerse aún más en cuenta en la profesión, así como particularmente en la docencia.

\section{NOTAS}

* Miembro del grupo de investigación Evaluación de la Calidad en Interpretación Simultanea (ECIS) de la Universidad de Granada (ref. HUM-560).

1. Para más información sobre los estudios interdisciplinares y específicos llevados a cabo en el ámbito de la entonación del intérprete, véase Barrango-Droege, Collados Aís y Pazos Bretaña (2011).

2. Grupo de investigación HUM560 (ECIS, Evaluación de la calidad de la interpretación simultánea), financiado por la Junta de Andalucía. Este grupo, con sede en la Universidad de Granada, realizó también investigaciones en diversos proyectos de investigación de carácter autonómico y nacional en los que también participaron miembros de otras universidades españolas.

3. La investigación se incluye en un proyecto más amplio del grupo ECIS en el que se realizaron en paralelo varias encuestas. Varias investigadoras administraron cuestionarios específicos sobre otros nueve parámetros de calidad. Los cuestionarios, si bien siguen una estructura común, están adaptados específicamente a cada uno de los parámetros considerados. 


\section{REFERENCIAS BIBLIOGRÁFICAS}

Ahrens, Barbara (2005): Prosodie beim Simultandolmetschen. Frankfurt am Main: Peter Lang. AIIC (2004): Practical guide for professional conference interpreters. Consultado el 11 de enero de 2015), <http://aiic.net/page/628/practical-guide-for-professional-conference-interpreters/ lang/1\#33>.

BüHLER, Hildegrund (1986): Linguistic (semantic) and extra-linguistic (pragmatic) criteria for the evaluation of conference interpretation and interpreters. Multilingua 5(4):231-235.

Barranco-Droege, Rafael, Collados Aís, Ángela, Pazos Bretaña, José Manuel (2011): Intonation. En: Ángela Collados Aís, Emilia Iglesias Fernández, E. Macarena Pradas MAcíAs et al., eds. Qualitätsparameter beim Simultandolmetschen. Interdisziplinäre Perspektiven. Tübingen: Narr Verlag, 61-92.

Cheung, Andrew K.F. (2013): Non-native accents and simultaneous interpreting quality perceptions. Interpreting 15(1):25-47.

Collados Aís, Ángela (1998): La evaluación de la calidad en interpretación simultánea. La importancia de la comunicación no verbal. Granada: Comares Interlingua.

Collados Aís, Ángela (2001): Efectos de la entonación monótona sobre la recuperación de la información en receptores de interpretación simultánea. Trans 5:103-110.

Collados Aís, Ángela (2007): La incidencia del parámetro entonación. En: Ángela Collados Aís, E. Macarena Pradas Macías, Elisabeth STÉvaux et al., eds. Evaluación de la calidad en interpretación simultánea: parámetros de incidencia. Granada: Comares, 159-174.

Collados Aís, Ángela (2008): Evaluación de la calidad en interpretación simultánea: contrastes de exposición e inferencias emocionales. Evaluación de la evaluación. En: Gyde Hansen, Andrew Chesterman, Heidrun Gerzymisch-Arbogast, eds. Efforts and Models in Interpreting and Translation Research: A tribute to Daniel Gile. Amsterdam/Philadelphia: John Benjamins, 193-214.

Collados Aís, Ángela (2009): Marco evaluador de la calidad en interpretación simultánea. En: Sonia Bravo Utrera, Rosario García López, eds. Estudios de traducción: perspectivas. Zinaida Lvoskaya in memoriam. Frankfurt am Main: Peter Lang, 145-169.

Collados Aís, Ángela (2010): La evaluación de la calidad en interpretación simultánea: pautas evaluadoras según usuarios. En: Rosa Rabadán, Trinidad Guzmán, Marisa Fernández, eds. Lengua, traducción, recepción. En honor de Julio César Santoyo. León: Universidad de León, 67-90.

Collados Aís, Ángela, Pradas Macías, E. Macarena, Stévaux, Elisabeth et al., eds. (2007): Evaluación de la calidad en interpretación simultánea: parámetros de incidencia. Granada: Comares.

Collados Aís, Ángela, Iglesias Fernández, Emilia, Pradas Macías, E. Macarena et al., eds. (2011): Qualitätsparameter beim Simultandolmetschen. Interdisziplinäre Perspektiven. Tübingen: Narr Verlag.

DARó, Valeria (1990): Voice frecuency in languages and simultaneous interpretation. The Interpreter's Newsletter 11:27-55.

Dejean Le FÉAL, Karla (1981): Lectures et improvisations. Incidences de la forme de l'énonciation sur la traduction simultanée (français-allemand). Fremdsprachen 1:29-32.

García Becerra, Olalla (2012): First Impressions in Interpreting Quality Assessment: The Incidence of Nonverbal Communication. En: Amparo Jiménez Ivars, María Jesús Blasco MaYor, eds. Interpreting Brian Harris. Recent Developments in Translatology. Frankfurt am Main: Peter Lang, 173-192.

GARzone, Giuliana (2003): Reliability of quality criteria evaluation in survey research. In: Ángela Collados Aís, Ma Manuela Fernández Sánchez, Daniel Gile, eds. La evaluación de la calidad en interpretación: investigación. Granada: Comares Interlingua, 23-30.

Gile, Daniel (1991): A communication-oriented analysis of quality in non literary translation and interpretation. En: Mildred E. Larson, ed. Translation: Theory and Practice. Tension and Interdependence. Binghamton NY: SUNY, 188-200. 
Herbert, Jean (1970): Manual del intérprete. Ginebra: Librairie de l’Université Georg.

Holub, Elisabeth (2010): Does intonation matter? The impact of monotony on listener comprehension. The Interpreter's Newsletter 15:117-126.

Iglesias Fernández, Emilia (2007): La incidencia del parámetro agradabilidad de la voz. En: Ángela Collados Aís, E. Macarena Pradas Macías, Elisabeth Stévaux et al., eds. Evaluación de la calidad en interpretación simultánea: parámetros de incidencia. Granada: Comares, 37-51.

Iglesias Fernández, Emilia (2011): Stimme. En: Ángela Collados Aís, Emilia Iglesias Fernández, E. Macarena Pradas Macías et al., eds. Qualitätsparameter beim Simultandolmetschen. Interdisziplinäre Perspektiven. Tübingen: Narr Verlag, 33-60.

Jiménez Ivars, Amparo (2011): Kohäsion. En: Ángela Collados Aís, Emilia Iglesias FernánDEZ, E. Macarena PradAs Macías et al., eds. Qualitätsparameter beim Simultandolmetschen. Interdisziplinäre Perspektiven. Tübingen: Narr Verlag, 173-189.

Katz, Daniel J. (1989): Pour un enseignement de l'expression orale dans les écoles d'interprètes. En: Laura Gran, John M. DodDs, eds. The Theoretical and Practical aspects of Teaching Conference Interpretation. Udine: Campanotto Editore, 217-218.

Kurz, Ingrid, Pöchнacker, Franz (1995): Quality in TV interpreting. Translatio- Nouvelles de la FIT- FIT Newsletter 15(3/4):350-358.

LLISTERRI, Joaquim (2014): Los elementos suprasegmentales. $<$ http://liceu.uab.es/ joaquim/phonetics/fon_prosod/suprasegmentales.html>.

MazetTi, Andrea (2005): The influence of segmental and prosodic deviations on source- text comprehension in simultaneous interpretation. The Interpreter's Newsletter 12:125-147.

NAFá WAASAF, M. Lourdes (2005): Análisis acústico-discursivo de la entonación en interpretación simultánea inglés británico-español peninsular. Aplicaciones a la didáctica y la investigación de lenguas. Tesis doctoral. Granada: Universidad de Granada.

Pradas Macías, E. Macarena (2006): Probing quality criteria in simultaneous interpreting. The role of silent pauses. Interpreting 8(1):25-43.

Russo, Mariachiara (2005): Simultaneous film interpreting and users' feedback. Interpreting 7(1):1-26.

Schweda Nicholson, Nancy (1992): Linguistic theory and simultaneous interpretation: Semantic and pragmatic considerations. Babel 38(2):90-100.

SHLesinger, Miriam (1994): Intonation in the production and perception of simultaneous interpretation. En: Sylvie LAMBERT, Barbara Moser-Mercer, eds. Bridging the Gap. Amsterdam/Philadelphia: Benjamins, 225-236.

StÉvaux, Elisabeth (2007): La incidencia del parámetro acento. En: Ángela Collados Aís, E. Macarena Pradas Macías, Elisabeth Stévaux et al., eds. Evaluación de la calidad en interpretación simultánea: parámetros de incidencia. Granada: Comares, 17-35.

Stévaux, Elisabeth (2011): Akzent. En: Ángela Collados Aís, Emilia Iglesias Fernández, E. Macarena Pradas Macías et al., eds. Qualitätsparameter beim Simultandolmetschen. Interdisziplinäre Perspektiven. Tübingen: Narr Verlag, 141-172. 